\title{
EFFECT OF SILVER NANOPARTICLES ON THE TREATMENT OF ODONTOGENIC- INDUCED ORO-FACIAL INFECTION IN RATS (HISTOLOGICAL AND IMMUNO-HISTOCHEMICAL STUDY)
}

\author{
Mervat Mohamed Youssef*
}

\begin{abstract}
The origin of odontogenic oro-facial infections is either periodontal infections or dental caries, which have expanded beyond the alveolar bone for the involvement of the facial spaces and face. An increasingly growing problem in treating infectious diseases is Multi-drug resistance (MDR). After the introduction of nanotechnology, silver nanpoarticles (AgNPs) have become immense antimicrobial agents. This investigation aimed to study the effect of AgNPs in treatment of Porphyromonas gingivalis- induced oro-facial infection in rats using routine histopathological evaluation and immuno-histochemical investigation of TNF- $\alpha$. Thirty adult male albino rats were used and divided into three equal groups. Group I: served as control. Group II: were infected once by intramuscular injection the cheek mucosa opposite to upper right first molar with $1 \times 10^{9} \mathrm{CFU} /$ $\mathrm{mL}$ of Porphyromonas gingivalis, and left for fourteen days. Group III: were subjected to the same procedure as group II then to daily injection at the site of infection with AgNPs (10 nm) with a concentration of $15 \mu \mathrm{g} / \mathrm{ml}$ of distilled water for fourteen days. At the end of the experiment, the animals were euthanized, samples were taken from the cheeks at the site of infection, processed and stained. The results revealed $P$. gingivalis- induced orofacial infection resulted in extensive tissues destruction as well as massive expression of TNF- $\alpha$. The treatment with AgNPs (10nm) resulted in marked improvement of the suppuration and regression in the inflammation as well as withdrawal of TNF- $\alpha$ expression. The author concluded that it is possible to employ AgNPs as wide spectrum antimicrobials against Multi- drug resistance.
\end{abstract}

KEYWORDS Odontogenic oro-facial infection, Porphyromonas gingivalis, Silver nanoparticles, Multi-drug resistance (MDR),

\section{INTRODUCTION}

Health issues are commonly caused by maxillofacial and oral infections. Besides, such infections are frequently the key reasons for dental consultation worldwide, they are either odontogenic or non odontogenic. Planells et al., stated that most orofacial infections are considered to be odontogenic in origin. Trauma, dental caries' sequelae or periodontal disease are the cause of them ${ }^{(1)}$ and can lead to significant complications, including osteomyelitis,

\footnotetext{
* Associate Professor, Oral Biology Department. Faculty of Dentistry, Suez Canal University.
} 
the infections of deep fascial space, and jaw's osteoperiostitis ${ }^{(2)}$ in case they are not managed in a timely and proper manner. The mortality and morbidity caused by such infections are dependent on involvement site and spread degree to other tissues. Further, for managing such infections in a scientific manner, disease's aetiology and pathology should be identified correctly. It is advisable to use the antimicrobials therapy soon after diagnosis but before surgery as it is able to shorten infection period and reduce related risks including bacteremia ${ }^{(3)}$.

In the majority of oro-facial infections, the predominant pathogens are likely represented by anaerobes, whereas microorganisms are the main cause of periodontal disease, gingivitis and dental caries. According to previous studies, Porphyromonas gingivalis ( $P$. gingivalis) is the key pathogen which is in charge of adult progressive periodontal disease ${ }^{(4,5)}$ and endodontic abscesses ${ }^{(6,7)}$. Such gram negative organism has a group of virulence factors such as gingipain, hemolysins, lipopolysaccharide, lectinlike adhesins, fimbriae, and hemagglutinins in addition to several proteolyticenzymes ${ }^{(4,8)}$.

Multi-drug resistance (MDR) is considered an increasing problem in treating infectious diseases where the broad-spectrum antibiotics which are widely used have generated antibiotic resistance to several human bacterial pathogens. Recent developments in nanotechnology have made great interest and focused huge research efforts on metallic silver nanoparticles' biomedical evaluation and revaluation. This is owing to their certain and genuine biological, chemical, and physical characteristics. In particular, such characteristics are perceived as non-traditional antimicrobial agents $^{(9,10)}$. Furthermore, Ag NPs' s impressive antimicrobial activity is considered a good starting point for developing and designing of nanosilverbased novel biomedical products including catheters $^{(11)}$, orthopedic materials and devices ${ }^{(12)}$, bandages, antiseptic sprays ${ }^{(13)}$. As a result of the intrinsic wide bactericidal effects of AgNPs shown against both Gram-positive and Gram-negative bacteria in addition to their physicochemical features, they represent one of the most commonly utilized metallic nanoparticles in contemporary antimicrobial applications ${ }^{(14)}$.

TNF (Tumor Necrosis Factor; cachectin or cachexin, which was once called TNF- $\alpha$ or Tumor Necrosis Factor alpha) represents a cytokine (a cell signaling protein) that is included in systemic inflammation. In addition, it is one of the cytokines making up the reaction of the acute case. Macrophages and T- lymphocytes and activated monocytes produce the TNF- $\alpha$, which is one of the primary components of the inflammatory response that results in damaging tissues. Furthermore, TNF- $\alpha$ is an important pro-inflammatory cytokine present $^{(15,16)}$.

This study aimed to examine the effect of silver nanoparticles in treatment of $P$. gingivalisinduced oro-facial infection in rats using routine histopathological examination and evaluate the inflammatory response through immunohistochemical localization of TNF $\alpha$ in the oral and skin tissues of the cheek.

\section{MATERIALS AND METHODS}

Animals and experimental design: Thirty adult male three months old albino rats 150- 180 gram body weight were used in this investigation obtained from Laboratory House of College of Veterinary Medicine and Animal Resources, Suez Canal University. The rats were acclimated for 10 days before starting experiment and under supervision of veterinarian. They maintained under clean, pathogen-free conditions in rat cages and labeled with numerical numbers in well ventilated animal house at the faculty of dentistry, Suez Canal University, at temperature of $27-30^{\circ} \mathrm{C}, 12 \mathrm{~h}$ natural light and $12 \mathrm{~h}$ darkness, with free access to tap water and dry rat pellet. They were divided randomly into three groups as follows:

Group I: consisted of 10 rats and served as normal negative control group. 
Group II: (positive control) consisted of 10 rats, they were infected once by intramuscular injection of the cheek mucosa at the vestibule opposite to upper right first molar with $1 \times 10^{9} \mathrm{CFU} / \mathrm{mL}$ of $P$. gingivalis and left for fourteen days ${ }^{(17)}$. P. gingivalis (gengsig® kit) was purchased from Primer Design, United kingdom. Then cultured and prepared in vacsera of the Holding Company for Biological Products and Vaccines in Giza, Egypt.

Group III: consisted of 10 rats, they were subjected to the same procedure as group II then to daily injection at the site of infection with silver nanoparticles $(10 \mathrm{~nm})$ with a concentration of $15 \mu \mathrm{g} /$ $\mathrm{ml}$ of distilled water for fourteen days ${ }^{(18)}$. Spherical silver nanoparticles were purchased from Egyptian Nanotechnology Company "Nano Gate", Nasr City.

The percentage of mortality for each group were calculated and recorded (no mortality cases encountered). Gross examination of head and neck of each animal was carried out through the whole experimental period.

At the end of the experiment, the animals of the different groups were euthanized by cervical dislocation.

Tissue processing and staining: Samples were taken from the cheek of all animals at the site of infection, fixed in $10 \%$ neutral buffered formalin, processed, embedded in paraffin wax and $4-5 \mu \mathrm{m}$ thick section were cut. Then they were stained with hematoxlyin and eosin for histopathological examination. For immuno-histochemical (IHC) evaluation of tumor necrosis factor Alpha, $5 \mu \mathrm{m}$ sections were cut and mounted on positively-charged slides. The immunostaining were performed using rabbit polycolonal antibody to $\mathrm{TNF} \alpha$, purchased from Gene Tex International Corporation, Cat. No. GTX110520. The steps of IHC were followed according to manufacturer's instructions. Stained tissue sections were photographed with Leica DM 1000 light microscopy and Camera using Leica Application Suite-LAS software in the Centre of Excellence of Molecular and Cellular Medicine (CEMCM), Suez Canal University.

\section{RESULTS}

\section{Clinical findings:}

Animals of group II were infected with $P$. gingivalis showed mucosal and/or cutaneous abscesses, accumulation of pus and development of fistulae (Figure1; A,B). Skin at the region of infection showed alopecia, redness and hotness. The rats showed signs of fatigue and loss of appetite. Lymphadenitis in the cervical region were encountered. While the animals of group III treated with silver nanoparticles for fourteen days showed marked improvement in the infected and inflamed tissues.

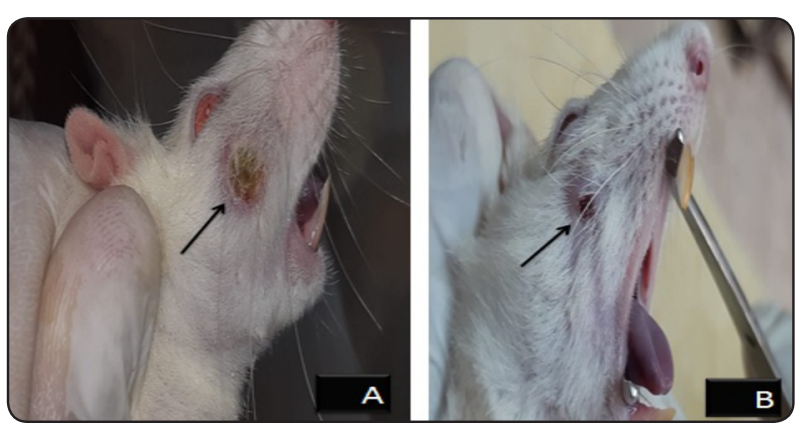

Fig.(1): A, B photomicrograph of the animal of group II showing cutaneous abscesses and fistulas (arrow). Skin at the region of infection showed alopecia, redness.

\section{Histopathological Results}

\section{Hematoxylin\& Eosin stains}

\section{1-Group I: (negative control ):}

Examination of the oral side of the cheek showing the epithelium is keratinized squamous with regular, broad, short and few rete ridges with lamina propria contains dense connective tissue. The submucosa is formed of strands of densely packed collagen fibers with loose areolar C.T. contain fat cells. Mixed buccal salivary glands were detected between the bundles of the buccinator muscles (Figure 2; A,B).

Skin side is composed of surface epithelium (epidermis) and lamina propria (dermis). The epithelium is keratinized stratified squamous epithelium type with few and short epithelial ridge. 


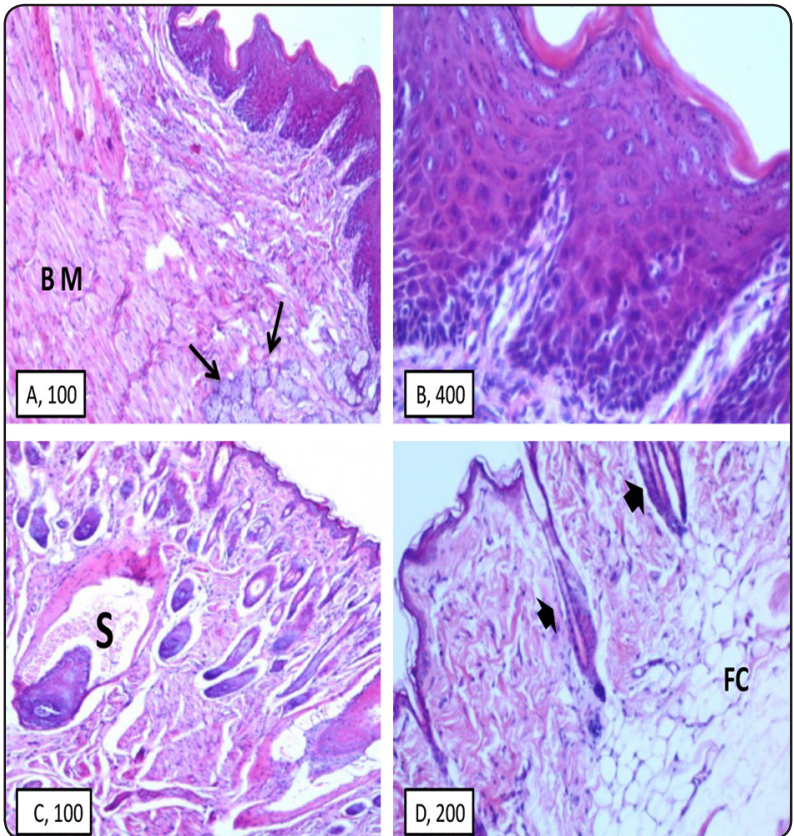

It consists of several strata of epithelial cells: stratum basal, spinosum, granulosum and cornium in addition to stratum lucidum. The dermis consists of C.T. formed of dense collagen bundles and elastic fibers, C.T. cells, fat cells, blood and neural elements in addition to skin appendages as hair, sebaceous and sweat glands (Figure 2; C,D).

\section{2- Group II: (positive control, P. gingivalis- in- duced oro-facial infection)}

Examination of the oro-facial tissues of the cheek of the infected rats with $P$. gingivalis for fourteen days showed massive inflammatory, necrotic/ apoptotic and degenerative changes that involved the surface epithelium and lamina propria of both oral and skin sides as well as the buccinators muscles and buccal salivary glands. The surface epithelium showed severe atrophic changes presented as a decrease in its thickness and loss of characteristic pattern of epithelial ridges which sometimes partially or completely absent. The epithelial cells presented edema, swelling, abnormal cytoplasmic vacuolization and/ or even apoptosis of some cells.

The lamina propria of both oral and skin sides revealed dissociation, degeneration of the collagen fibers with apparent decrease in the number of
Fig.(2): A,B) Photomicrographs of the buccal mucosa of the negative control rats showing surface epithelium of the keratinized stratified squamous type with regular, broad, few and short epithelium ridges toward the underlying lamina propria. The lamina propia and submucosa formed of densely packed collagen fibers and buccal salivary glands (arrows) are found between the bundles of the buccinator muscles (BM) (H\&E, ori. mag. 100, 400). C,D) Photomicrographs of the skin side of the cheek of the same group showing epidermis, dermis and hypodermis. Skin appendages as hair follicles (arrow heads), sebaceous (S) and sweat glands were seen. Underlying hypodermis showing a lot of fat cells (FC) (H\&E, ori. mag.100, 200).

fibroblasts, engorgement of blood in massive dilated blood vessels and massive inflammatory cell infiltration. The underling buccinator muscles bundles presented with massive degeneration and necrosis with extensive hemorrhagic areas and extreme inflammatory cell infiltration, in addition to loss of organization of the remaining degenerated bundles. Degeneration and cystic transformation of buccal salivary glands cannot be neglected. (Figure3).

\section{3- Group III: (P. gingivalis- induced oro-facial in- fection treated with silver nanoparticles)}

The oro-facial tissues of the cheek of rats treated with daily injection at the site of infection with silver nanoparticles $(10 \mathrm{~nm})$ for fourteen days showed marked decreased injury, necrosis /apoptosis, remarkable improvement and regeneration of the destroyed tissues. The buccal mucosa and skin showed nearly the same histological feature of the negative control group. Almost complete regeneration of its cellular, vascular and neural elements. The surface epithelium revealed increase in the thickness and numbers cells as well as increase in the length of the epithelial ridges. 
The lamina propria showed marked increase in the cellularity and density of the collagen fibers which became highly organized. The buccal salivary gland showed marked improvement in the histological structure of their acini and ducts.
The buccinators muscles bundles became more organized and integrated. Marked improvement of the suppuration and regression in the inflammatory cell infiltration were obvious (Figure 4).

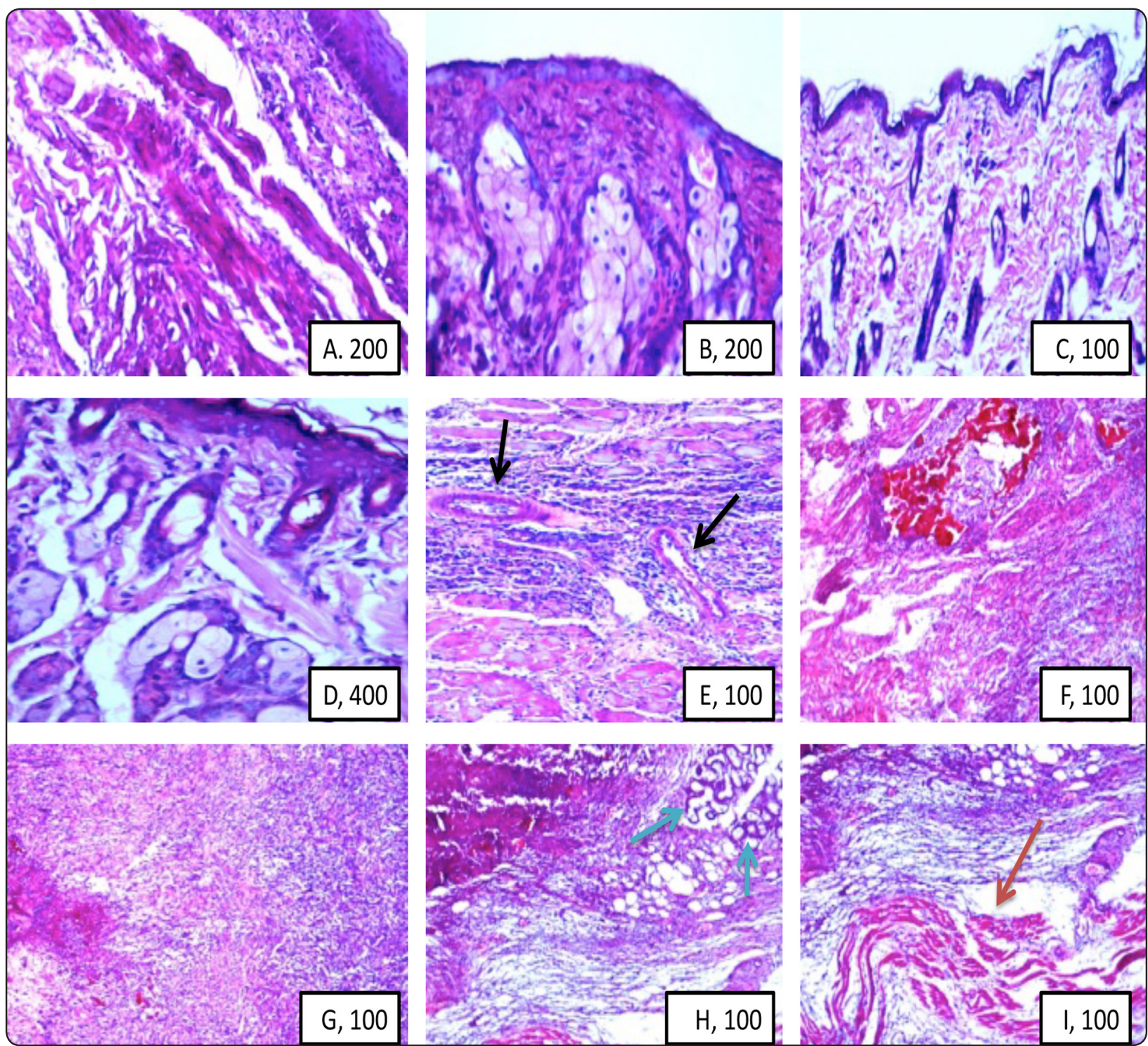

Fig.(3): Photomicrographs of tissues of the oral and skin sides of the cheeks of rats were infected with P. gingivalis for fourteen days. (A.B.C,D) showing the surface epithelium revealed severe atrophy and degeneration, loss of characteristic pattern of rete ridges. The epithelial cells presented edema, swelling, abnormal cytoplasmic vacuolization and necrosis/ apoptosis. (A,C,D) showing the lamina propria of both sides presented dissociation, degeneration of the collagen fibers and C.T. cells. (E,F,G,H,I) showing engorgement of blood in massive dilated blood vessels (black arrows) and massive inflammatory cell infiltration. The buccinator muscles bundles presented with massive degeneration, necrosis, extensive hemorrhagic areas and extreme inflammatory cell infiltration in addition to loss of organization of the remaining degenerated bundles (red arrows) Degeneration and cystic transformation of buccal salivary glandswere seen (blue arrow) (H\&E, ori. mag.100, 200,400). 


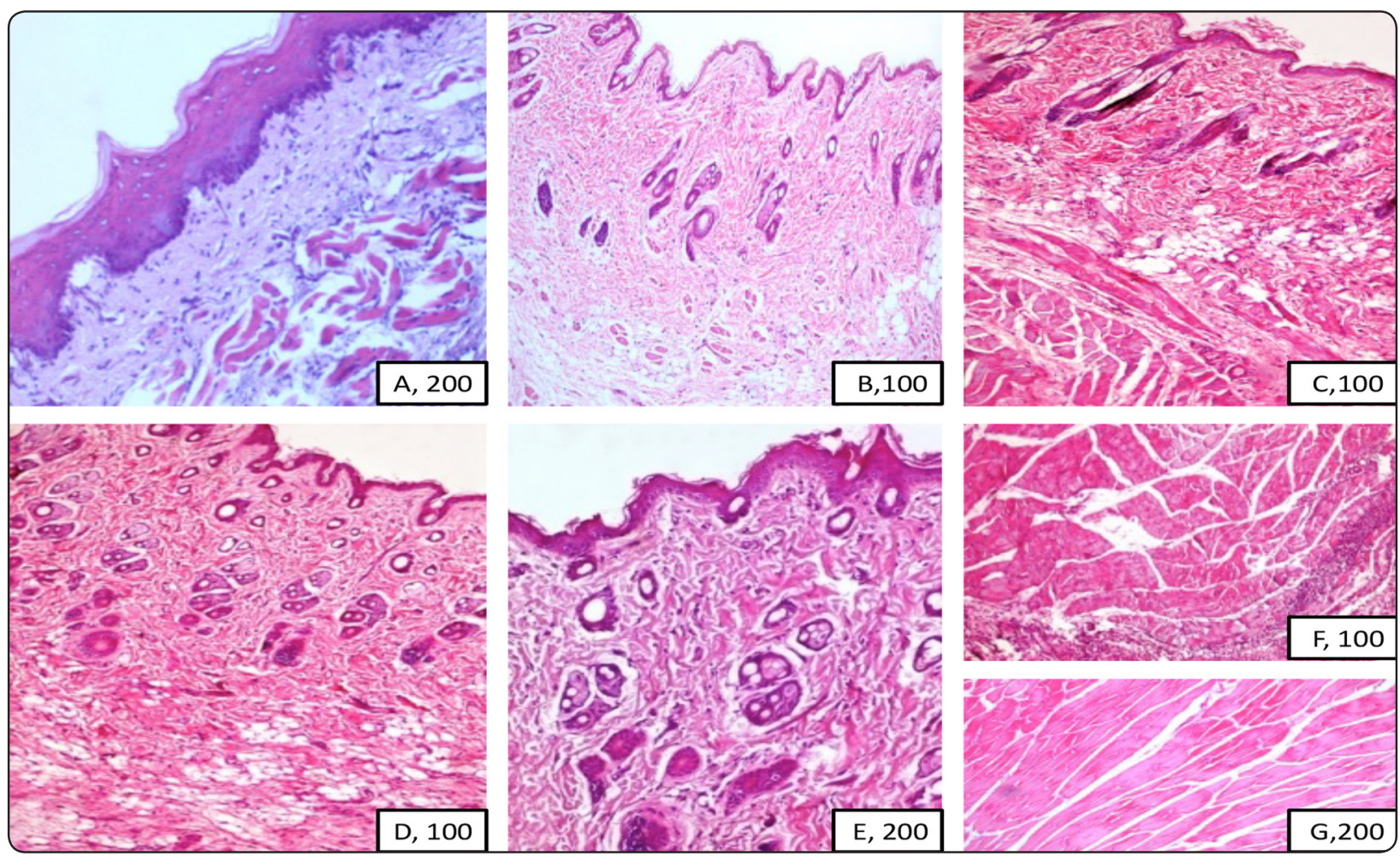

Fig.(4): Photomicrographs of the oro-facial tissues of the cheeks of rats treated with daily injection at the site of infection with silver nanoparticles $(10 \mathrm{~nm})$ for fourteen days showing $(\mathrm{A})$ the buccal mucosa increased thickness of the surface epithelium with elongation of its epithelial ridges. The lamina propria showing increase in the cellularity, normal density and organization of the collagen fibers with remarkable regression in the inflammatory cell infiltration. (B,C,D,E) showing improvement and regeneration in the histological structure of the epidermis, dermis and skin appendages. (F,G) showing the buccinators muscles bundles became more organized and integrated with marked improvement of the suppuration and regression in the inflammatory cell infiltration (H\&E, ori. mag.100, 200).

\section{Immuno-histochemical localization of TNF- $\alpha$}

Examination of the pro- inflammatory cytokine TNF- $\alpha$ expression (brown staining) in normal control tissue revealed weak expression localized to the epithelium, lamina propria, dermal, hypodermal layers as well as buccinators muscles bundles (Fig.5; A).While samples infected with P.gingivalis for fourteen days showed massive expression of TNF- $\alpha$ in all around above mentioned tissues particularly at the areas of severe inflammation, tissue destruction (Fig.5; B,C,D). Samples treated with daily injection at the site of infection with silver nanoparticles $(10 \mathrm{~nm})$ for fourteen days showed nearly completely withdraw expression of TNF- $\alpha$ in the oral and skin tissues of the cheek (Fig.5; E,F).

\section{DISCUSSION}

The origin of odontogenic oro-facial infections is either periodontal infections or dental caries, which have expanded beyond the alveolar bone for the involvement of the facial spaces surrounding the oral cavity and face. In addition, such infections have tendency towards spreading along the planes of least resistance generated by the affected tooth's supporting structures ${ }^{(19)}$. Severe infections of odontogenic origin frequently involve a complex polymicrobial mix of aerobes, facultative aerobes and strict anaerobes working together ${ }^{(20)}$.

In the present study, the rats infected once by deeply injecting the buccal mucosa of the vestibule 


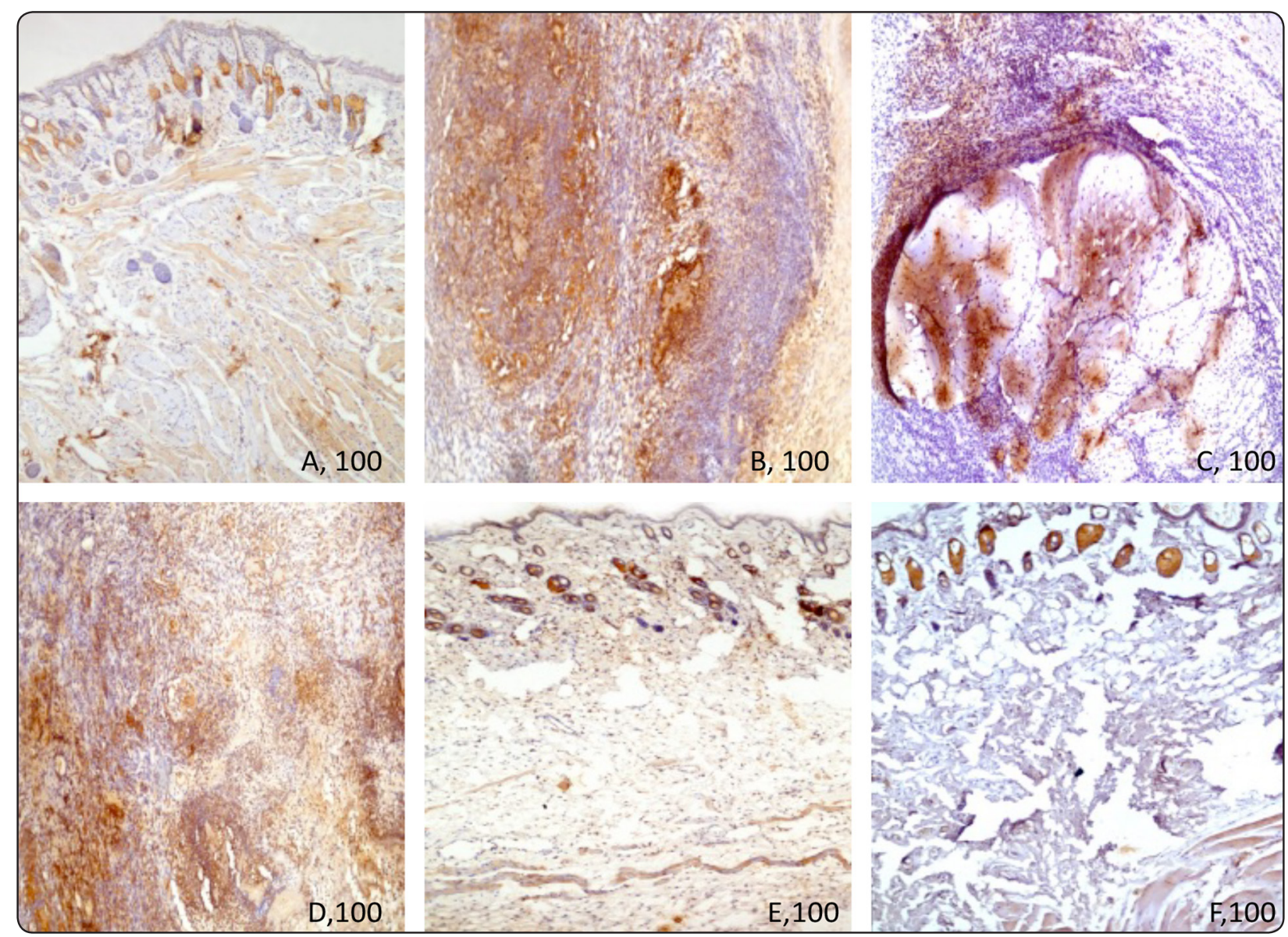

Fig.(5): Photomicrographs the oro-facial tissues of the cheek of all groups showing different staining reactivity to the polycolonal antibody to TNF- $\alpha$, for detect TNF alpha pro- inflammatory protein. A- control group. B,C,D- P. gingivalis- infected group. E,F- nanosilver treated group (orig. mag.100).

opposite to upper right first molar with $1 \times 10^{9}$ $\mathrm{CFU} / \mathrm{mL}$ of $P$. gingivalis and left for fourteen days coincided with that presented by Han et al., ${ }^{(17)}$ and How et al., (21) who stated that $P$. gingivalis, which is one of the Gram-negative anaerobic bacteria, is the key etiologic agent that has contributions to chronic periodontitis and periodontal bone resorption. Together with Chow et al., ${ }^{(19)}$ who reported that the affected tooth's location is able to predict the path of odontogenic orofacial infection's spread. This is agreement with our clinical finding that registered signs of inflammation, mucosal or cutaneous abscesses and accumulation of pus and fistulas at the oral and/ or skin sides of cheek opposite to the site of injection .
The histopathological and innuno-histochemical results of the present study demonstrated massive inflammatory, necrotic/ apoptotic and degenerative changes as well as heavy expression of TNF- $\alpha$ that involved the oral and facial tissues of the infected cheek with P.gingivalis and left for fourteen days. The animals displayed characteristic features of tissue destruction, including extensive partially confluent purulent foci of extensive necrosis and apoptosis. Our result concur with Nonnenmacher and others ${ }^{(22)}$ who stated that the anaerobic bacteria pathogen $P$. gingivalis is the cause of several conditions of chronic inflammatory disease of soft and hard tissues reinforcing the teeth, where the tooth could be lost in severe cases. Furthermore, Shweta 
and Prakash ${ }^{(23)}$ reported a microbiological review about dental abscess and stated that in the cases of the dentoalveolar abscess and chronic endodontic infection, the commonly detected pathogens are $P$. endodontalis and $P$. gingivalis.

The results of current study support and extend the results of previous studies of Andrian and coworkers $^{(24)}$ who found that cysteine proteinase gingipains, which are essential virulence factors contributing to periodontitis's pathogenesis and degrading extracellular matrix proteins including fibronectin, laminin, and type IV collagen in addition to matri-gel protein constituents, are produced by $P$. gingivalis. Furthermore, they indicated that when cytokines (IL-1, IL-6, IL-8, and TNF- $\alpha$ ) are excessively produced in inflamed periodontal tissues, they were suggested to be the cause of periodontitis progression by releasing the tissue-derived enzymes, host matrix metalloproteinases, that destroy the extracellular matrix and bone.

Despite the fact that, the core of therapy is the pus's surgical drainage and dental extraction, there is a strong need for antibiotic treatment for preventing infection's spread and its complications ${ }^{(19)}$. Repeated application of antibiotics to treat infection increases the resistance of organisms and so the routine antibiotic prophylaxis become ineffective. Previous study done by Bernan et al., ${ }^{(25)}$ demonstrated that silver nanoparticles have an effective antibacterial impact and have been widely utilized in medicine. This go with our histopathological and immuo-histochemical investigation that showed marked decreased injury, necrosis /apoptosis with remarkable improvement and regeneration of the destroyed oral and cutaneous tissues of the cheek of rats treated with daily injection at the site of infection with silver nanoparticles $(10 \mathrm{~nm})$ for fourteen days.

The topical application of silver nanoparticles coincided with Liao et al. ${ }^{(18)}$ to overcome systemic cytotoxicity of the drug. Our experiment coincided with previous study done by Lu et al., ${ }^{(26)}$ who made a preparation of different sizes of AgNPs $(\sim 5,15$ and
$55 \mathrm{~nm}$ ) for displaying silver nanoparticles' size-dependent antibacterial activities against oral anaerobic pathogenic bacteria. Their results indicated that AgNPs $(5 \mathrm{~nm})$ displayed the strongest antibacterial activity against oral bacteria.

In our opinion, the marked improvement of the suppuration, regression in the inflammatory cell infiltration as well as withdrawal of TNF- $\alpha$ expression confirm that silver nanoparticles have the ability to destroy several drug-resistant strains and stop biofilm formation. Consequently, they showed a significant ability in antibacterial application. Therefore, silver nanoparticles are perceived as an alternative approach to antimicrobial therapy. This consistent with Franci et al., ${ }^{(27)}$.Yuan et al., ${ }^{(28)}$, Ibraheem et al., ${ }^{(29)}$.

One of the interesting findings in this study is the reduction of $P$. gingivalis colonization manifested by the massive reduction in the inflammatory reaction after injection of the infected areas with $\mathrm{Ag}$ nanoparticles. This is supported by previous studies hypothesizing that nanosilver-based systems have two phenomena to practice their antimicrobial influences as follows: first, the damage of microbial membrane through attaching AgNPs physicochemically on the cell surface and the following functional and structural alterations (including cytoplasm leakage, membrane piercing, gap formation, and membrane destabilization). Second, the damage of microbial sub-cellular structure through releasing free $\mathrm{Ag}+$ ions and the following ROS (Reactive Oxygen Species) and free-radical generation (hypochlorous acid, singlet oxygen, hydroxyl radical, hydrogen peroxide, and superoxide anion), or the inactivation of essential macromolecule (enzymes, proteins, and nucleotides) ${ }^{(30-32)}$.

Moreover, the results of previous studies indicated that AgNPs' antibacterial impact on Gram-negative bacteria was stronger compared to Gram-positive bacteria. A good explanation of this phenomenon is the existent difference of the thickness of cell wall between Gram-negative bacteria (7-8 $\mathrm{nm})$ and 
Gram-positive bacteria (20-80 nm) as the cell wall of gram-positive bacteria is a relatively thick consisting of numerous peptidoglycan layers that serve as a barricade to the penetration of $\mathrm{Ag}^{+}$ions into the cytoplasm. On the contrary the cell wall of gramnegative is thin consisting of a peptidoglycan layer, and therefore, the cell wall can be easily damaged by $\mathrm{Ag}^{+}$ions $^{(33,34)}$.

Our results are in agreement with several previous studies demonstrated that AgNPs are very effective in killing MDR bacteria (Multi-drug Resistant bacteria) by intervening into the mechanisms of their defense. In addition, they are utilized alone or in conjunction with antibiotics ${ }^{(35-37)}$.

In summary, $P$. gingivalis induced orofacial infection resulted in massive tissues destruction. In general, microorganisms gain resistance to antibiotics by repeating antibacterial therapy course. In treating infectious diseases, the Multi-drug resistance (MDR) represents an increasing problem. After introducing nanotechnology, there emerged an urgent need for developing an alternative strategy. The results obtained within this study highlight that AgNPs are remarkably antimicrobial agents. AgNPs are able to have interaction with $P$. gingivalis microorganism and affect both growing and mature bacterial biofilms. That's why, they can be utilized as wide spectrum antimicrobials. Their antibacterial impact seems to be generated by the increased surface area and their ultra-small size. Through both of these mechanisms, the AgNPS are able to destroy the membrane and then they cross microbe's body and create intracellular damage.

From our point of view, the most important clinical and therapeutic options in the future are the AgNPs due to their bactericidal impact, particularly due to the lack of novel antimicrobials agents against the emergent MDR microorganisms, therefore AgNPs may act as powerful tool against multidrug-resistance.

In conclusion, odontogenic oro-facial infections emerge either from periodontal infections or den- tal caries. The MDR (Multi-drug Resistance) is an increasing problem in treating infectious diseases. Following the introduction of nanotechnology, the AgNPs have become noticeable antimicrobial agents as they are able to have interactions with numerous microorganisms and affect their growth. Consequently, they can be utilized as wide spectrum antimicrobials against MDR bacteria.

\section{REFERENCES}

1. Planells, d.P., Barra S.M. and Santa, E.T.: Antibiotic prophylaxis in pediatric odontology. An update. Med. Oral Patol. Oral Cir. Bucal. 2006;11:352-357.

2. Al-Malik, M. and Al-Sarheed, M.: Pattern of management of oro-facial infection in children: A retrospective. Saudi J Biol Sci. 2017; 24: 1375-1379.

3. Sandor, G.K; Low, D.E; Judd, P.L. and Davidson, R.J.: Antimicrobial treatment options in the management of odontogenic infections. J Can Dent Assoc. 1998;64:508-14.

4. Xu, W.; Zhou, W.; Wang, H. and Liang, S.: Roles of Porphyromonas gingivalis and its virulence factors in periodontitis. Advances in Protein Chemistry and Structural Biology. 2020; 120: Pages 45-84

5. Griffen, A.L; Becker, M.R; Lyons, S.R; Moeschberger, M.L and Leys, E.J: Prevalence of Porphyromonas gingivalis and periodontal health status. J Clin Microbiol. 1998; 36:3239-42.

6. Siqueira ,J.F.; Rocas, I.N. and Silva, M.G.: Prevalence and Clonal Analysis of Porphyromonas Gingivalis in Primary Endodontic Infections. J Endod. 2008 ;34:1332-1336.

7. Siqueira, J.F and Rocas, I.N: Microbiology and treatment of acute apical abscesses. Clin Microbiol Rev. 2013; 26: 255-73.

8. Gibson, F.C. $3^{\text {rd }}$ and Genco, C.A.: Prevention of Porphyromonas gingivalis-induced oral bone loss following immunization with gingipain R1. Infect Immun. 2001; 69: 7959-63.

9. Alexander, J.W.: History of the medical use of silver. Surg. Infect. 2009; 10: 289-292.

10. Geraldo, D.A.; Needham, P.; Chandia, N.; Arratia-Perez, R.; Mora, G.C.; Villagra, N.A.: Green synthesis of polysaccharides-based gold and silver nanoparticles and their promissory biological activity. Biointerface Res. Appl. Chem. 2016; 6: 1263-1271. 
11. Thomas, R.; Soumya, K.R.; Mathew, J.; and Radhakrishnan, E.K. Inhibitory effect of silver nanoparticle fabricated urinary catheter on colonization efficiency of coagulase negative staphylococci. J. Photochem. Photobiol. B. 2015; 149: 68-77.

12. Burdușel, A.C.; Gherasim, O.; Grumezescu, A.M.; Mogoantă, L.; Ficai, A. and Andronescu, E.: Biomedical Applications of Silver Nanoparticles: An Up-to-Date Overview. Nanomaterials (Basel). 2018; 8: 681.

13. Durán, N.; Durán, M.; de Jesus, M.B.; Seabra, A.B.; Fávaro, W.J. and Nakazato, G.: Silver nanoparticles: A new view on mechanistic aspects on antimicrobial activity. Nanomed. Nanotechnol. Biol. Med. 2016; 12: 789-799.

14. Wang, L.; Hu, C. and Shao L.: The antimicrobial activity of nanoparticles: present situation and prospects for the future. Int J Nanomedicine. 2017;12:1227-1249.

15. Olsen, N.J. and Stein, C.M.: New drugs for rheumatoid arthritis. N Engl J Med. 2004; 350: 2167-79.

16. Hehlgans, T. and Pfeffer, K.: The intriguing biology of the tumor necrosis factor/tumour necrosis factor receptor superfamily: players, rules and the games. Immunology. 2005; 115: 1-20.

17. Han, X.; Lin, X.; Yu, X.; Lin, J; Kawai, T.; LaRosa, K. B. and Taubmana, M.A.: Porphyromonas gingivalis Infection-Associated Periodontal Bone Resorption Is Dependent on Receptor Activator of NF- $x \mathrm{~B}$ Ligand. Infect Immun. 2013; 8: 1502-1509.

18. Liao, C.; Li, Y. and Sie Chin Tjong, S.T.: Bactericidal and Cytotoxic Properties of Silver Nanoparticles. Int. J. Mol. Sci. 2019, 20: 449.

19. Chow, A.W.; Mandell, G. L.; Bennett, J. E. and Dolin, R.: Infections of the oral cavity, neck and head. Principles and practice of infectious diseases. 2010. pp. 855-71.

20. Robertson, D. and Smith, A. J.: The microbiology of the acute dental abscess. J Med Microbiol. 2009: 58: 155-162.

21. How, K.Y.; Song, K. Pand Chan, K.G.: Porphyromonas gingivalis: An Overview of Periodontopathic Pathogen below the Gum Line. Front Microbiol. 2016;7: 53.

22. Nonnenmacher, C.; Mutters, R. and Flores De Jacoby L.: Microbiological characteristics of subgingival microbiota in adult periodontitis, localized juvenile periodontitis and rapidly progressive periodontitis subjects. Clin Microbiol Infect. 2001;7:213-217.
23. Shweta and Prakash, S.K.: Dental abscess: A microbiological review. Dent Res J (Isfahan). 2013;10: 585-91.

24. Andrian, E.; Grenier, D. and RouabhiaIn, M.: Vitro Models of Tissue Penetration and Destruction by Porphyromonas gingivalis. Infection and immunity. 2004, 72: 4689-4698.

25. Brennan, S.A.; Fhoghlú, C.N.; Devitt, B.; O’Mahony, F.J.; Brabazon, D. and Walsh, A.: Silver nanoparticles and their orthopaedic applications. Bone Joint J. 2015; 97:582-589.

26. Lu, Z.; Rong, K.; Li, J.; Yang, H. and Chen, R.: Sizedependent antibacterial activities of silver nanoparticles against oral anaerobic pathogenic bacteria. J. Mater. Sci. Mater. Med. 2013; 24: 1465-1471.

27. Franci, G.; Falanga, A.; Galdiero, S; Palomba, L; Rai, M.; Morelli, G. and Galdiero, M.: Review Silver Nanoparticles as Potential Antibacterial Agents Molecules 2015; 20: 8856-8874.

28. Yuan, Y.G.; Peng, Q.L. and Gurunathan, S.: Effects of silver nanoparticles on multiple drug-resistant strains of Staphylococcus aureus and Pseudomonas aeruginosa from mastitis-infected goats: an alternative approach for antimicrobial therapy. Int J Mol Sci. 2017;18:569.

29. Ibraheem, S.A.; Kadhem, H.A.; Hadeethi, S.A.; Jabir, M.S.; Grigore, R.; Popa, M.; Gheorghe, I. and Floein, M.D.: Effects of silver nanoparticles on nosocomial Pseudomonas aeruginosa strains - an alternative approach for antimicrobial therapy. Rom Biotechnol Lett. 2019; 24: 286-293.

30. Kim, T.; Braun, G.B.; She, Z.G.; Hussain, S.; Rouslahti, E. and Sailor, M.J.: Composite porous silicon-silver nanoparticles as theranostic antibacterial agents. ACS Appl Mater Interfaces. 2016; 8: 30449-30457.

31. Seong, M. and Lee, D.G.: Silver nanoparticles against Salmonella enterica serotype typhimurium: role of inner membrane dysfunction. Curr Microbiol. 2017;74:661-670.

32. Zhao, R.; Lv, M.; Li, Y.; Sun, M.; Kong, W., Wang, L., Song, S.; Fan, C.; Jia, L.; Qiu, S.; Sun, Y.; Song, H. and Hao, R.: Stable nanocomposite based on PEGylated and silver nanoparticles loaded graphene oxide for longterm antibacterial activity. ACS Appl Mater Interfaces. 2017;9:15328-15341.

33. Chatterjee, T.; Chatterjee, B.K.; Majumdar, D.; Chakrabarti, P.: Antibacterial effect of silver nanoparticles and the modeling of bacterial growth kinetics using a modified Gompertz model. Biochim Biophys Acta. 2015;1850:299-306. 
34. Slavin, Y.N.; Asnis, J.; Hafeli, U.O. and Bach, H.: Metal nanoparticles: Understanding the mechanisms behind antibacterial activity. J. Nanobiotechnol. 2017;15:65.

35. Baptista, P.V.; McCusker, M.P.; Carvalho, A.; Ferreira, D.A.; Mohan, N.; Martins, M. and Fernandes, A.R.: Nanostrategies to fight multidrug resistant bacteria_- "A battle of the titans" Front. Microbiol. 2018;9:1441.
36. Katva, S.; Das, S.; Moti, H.S.; Jyoti, A. and Kaushik, S.: Antibacterial synergy of silver nanoparticles with gentamicin and chloramphenicol against Enterococcus faecalis. Pharmacogn. Mag. 2018;13:S828-S833.

37. Gurunathan, S.; Choi, Y.Z.; Kim, J.H.: Antibacterial efficacy of silver nanoparticles on endometritis caused by Prevotella melaninogenica and Arcanobacterum pyogenes in dairy cattle. Int. J. Mol. Sci. 2018;19:1210. 